\title{
ENDOSCOPIC EAR SURGERY; DOES IT HAVE AN IMPACT ON QUALITY OF LIFE
}

\author{
Ms. Virangna Taneja, Ms Rachel Easto, Mr Arunachalam Iyer
}

\section{Introduction}

Endoscopic ear surgery is a new mode of treatment but none of the studies have assessed the benefit to the quality of life of patients.

This study uses the validated Glasgow Benefit Inventory ( $\mathrm{GBI}$ ) to quantify the changes in quality of life.

\section{Material and method}

152 consecutive patients (operated by endoscopic, microscopic as well as combined route) were enrolled in the study from December 2013 till December 2015

The common indication were hearing loss and chronically discharging ear

\section{Results}

The response rate was $89.47 \%$ (no response -15 , deceased - 1).

There was considerable difference in terms of GBI score between endoscopic cases vs. others and social score was significantly higher in endoscopic cases ( $p$ value 0.045 )

Comparing the type of surgeries separately - Total, physical and social score were better in patients operated for Tympano/myringo by endoscopic route but were not significant.

Only social scores were better in patients operated for mastoid/stapes by endoscopic route.

In Stapes group there was improvement in AC, BC and A-B gap postop and it was significant ( $p$ value 0.00 for all three).

In endoscopic group-Mean improvement was $45.5 \%$ in $A C, 17.62 \%$ in $B C$ and $68.9 \%$ in A-B gap.

Improvement was $53.58 \%$ in $\mathrm{AC}$, $52.3 \%$ in $B C, 54.78 \%$ in $A-B$ gap in combined case.

Chorda was sacrificed in 1 case, displaced in 24 and was partially damaged in 3 cases.

\section{Conclusion}

This paper is the first to demonstrate correlation of quality of life in Endoscopic ear surgeries vs open and combined approach measured by the GBI

\begin{tabular}{|l|c|c|c|}
\hline & $\begin{array}{c}\text { Endoscopic } \\
\text { surgery (96) }\end{array}$ & $\begin{array}{c}\text { Combined } \\
\text { surgery (26) }\end{array}$ & $\begin{array}{c}\text { Open/microscopic } \\
\text { surgery (14) }\end{array}$ \\
\hline Total Score & 28.91 & 14.3 & 16.85 \\
\hline General Score & 30.43 & 13.6 & 21.34 \\
\hline Physical Score & 24.82 & 9.6 & 22.60 \\
\hline Social Score & 23.08 & 7.04 & -7.12 \\
\hline
\end{tabular}

Table 1 - GBI score results

\begin{tabular}{|l|c|c|c|}
\hline & $\begin{array}{c}\text { Endoscopic } \\
\text { surgery (28) }\end{array}$ & $\begin{array}{c}\text { Combined } \\
\text { surgery (1) }\end{array}$ & $\begin{array}{c}\text { Open/microscopic } \\
\text { surgery (0) }\end{array}$ \\
\hline Total Score & 47.23 & 66.5 & \\
\hline General Score & 50.92 & 100 & \\
\hline Physical Score & 36.35 & 100 & \\
\hline Social Score & 28.55 & -100 & \\
\hline
\end{tabular}

Table 2 - GBI score of stapes surgery

\begin{tabular}{|l|c|c|c|}
\hline & $\begin{array}{c}\text { Endoscopic } \\
\text { surgery (47) }\end{array}$ & $\begin{array}{c}\text { Combined } \\
\text { surgery (2) }\end{array}$ & $\begin{array}{c}\text { Open/microscopic } \\
\text { surgery (1) }\end{array}$ \\
\hline Total Score & 29.05 & 29.15 & -31 \\
\hline General Score & 29.8 & 35.4 & -29 \\
\hline Physical Score & 29.41 & 16.65 & 0 \\
\hline Social Score & 21.98 & 8.3 & -66.5 \\
\hline
\end{tabular}

Table 3 - GBI score - Tympano/myringo

\begin{tabular}{|l|c|c|c|}
\hline & $\begin{array}{c}\text { Endoscopic } \\
\text { surgery (21) }\end{array}$ & $\begin{array}{c}\text { Combined } \\
\text { surgery (23) }\end{array}$ & $\begin{array}{c}\text { Open/microscopic } \\
\text { surgery (13) }\end{array}$ \\
\hline Total Score & 4.16 & 10.75 & 20.53 \\
\hline General Score & 5.75 & 7.95 & 25.21 \\
\hline Physical Score & -0.81 & 5.06 & 24.35 \\
\hline Social Score & 18.24 & 11.59 & -2.55 \\
\hline
\end{tabular}

Table 4 - GBI score- Mastoid

\begin{tabular}{|l|c|c|c|c|}
\hline & \multicolumn{2}{|c|}{$\begin{array}{c}\text { Endoscopic } \\
\text { surgery (28) }\end{array}$} & \multicolumn{2}{c|}{$\begin{array}{c}\text { Combined } \\
\text { surgery (1) }\end{array}$} \\
\hline & pre-op & post-op & pre-op & post-op \\
\hline General Score & 60.55 & 32 & 52.5 & 24.37 \\
\hline Physical Score & 30.69 & 23.72 & 26.25 & 12.5 \\
\hline Social Score & 29.86 & 8.28 & 26.25 & 11.82 \\
\hline
\end{tabular}

Table 5 - Stapes Hearing Results
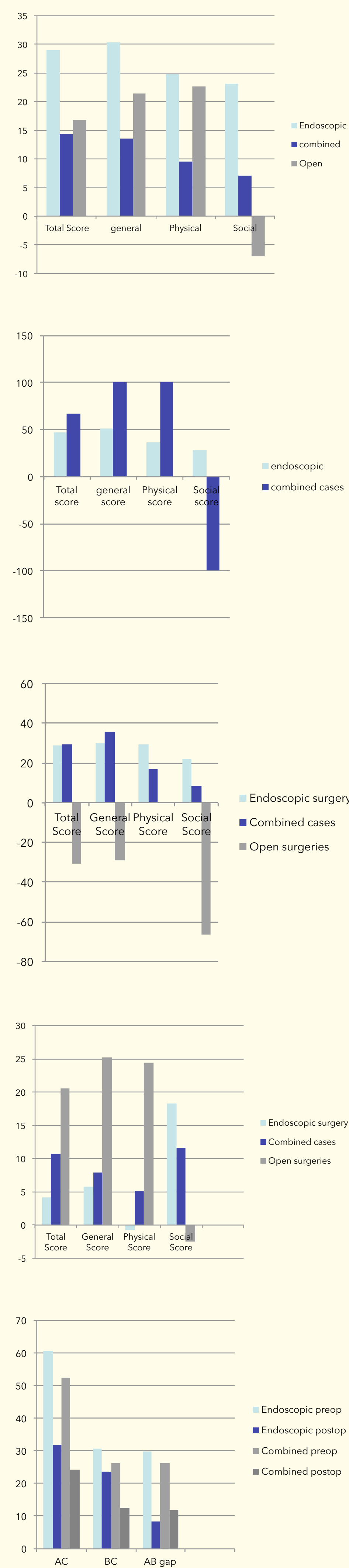